\title{
Biosensor Development with a Glutamate Receptor Ion-Channel Reconstituted in a Lipid Bilayer
}

\author{
Masayuki Uto*, Elias K. Michaelis**, Ing F. HU***, Yoshio Umezawa* and Theodore Kuwana*** \\ *Department of Chemistry, Faculty of Science, Hokkaido University, Sapporo 060, Japan \\ **Center for Biomedical Research, University of Kansas, Lawrence, KS 66046, USA \\ ***Center for Bioanalytical Research, University of Kansas, Lawrence, KS 66046, USA
}

\begin{abstract}
A glutamate receptor ion channel (RIC) protein, isolated and purified from rat brains, was reconstituted into artificial bilayer lipid membranes. This RIC protein was found to serve as a recognition site for a sensitive detection of L-glutamate. Two types of RIC sensors were tested. When the RIC was reconstituted as a single protein in a patchclamp membrane configuration, digital "off/on" signals were obtained for L-glutamate. With multiple proteins as a multi-channel sensor, the integrated dc signals were related to the glutamate concentration. The results from these two configurations are further discussed in terms of signal amplification and concentration dependence of the sensor.
\end{abstract}

Keywords Glutamate receptor ion channel, biosensor, single-channel sensor, multi-channel sensor

The possibility of creating new biosensors that are highly sensitive and selective, based on naturally occurring receptor ion-channels, has challenged several recent efforts. ${ }^{1-6}$ Notable among these are the reports by Yager $^{1,2}$ on the concept of biosensors from membrane proteins reconstituted in polymerized lipid bilayers and by Gotoh et al. ${ }^{3}$, Eldefrawi et al. ${ }^{4}$, Smith $^{5}$, and Taylor $e t$ $a l^{6}$ on the best studied neurotransmitter protein, the nicotinic acetylcholine $(\mathrm{ACh})$ receptor ion-channel (RIC). The actual observation of analytical results for a sensor demonstrating concentration dependence and taking advantage of the ion-channel amplification has been limited. To date, none have been reported for a purified glutamate RIC protein which had been reconstituted into a lipid bilayer structure.

In this paper, preliminary results for the verification of RIC responses from purified glutamate binding protein reconstituted in a lipid bilayer are reported with emphasis on the characteristics of the responses and the extent of amplification. The concentration dependence of the responses in both the single- and multi-channel configurations is discussed, although we acknowledge the limited nature of the current data. The difficulty of achieving active RIC with long-lived stability reconstituted into a lipid bilayer for any extensive concentration study remains a challenge. On the other hand, the ability to approach single-molecule detection selectively with a nearly real-time response is indeed tantalizing. The results reported herein verify that the purified and reconstituted GBP retains its biological function and, as such, may ultimately serve as an active site for a receptor-based biosensor.

\section{Experimental}

\section{Reagents and apparatus}

Phosphatidylcholine (PC), phosphatidylethanolamine (PE), phosphatidylseline (PS), cholesterol (Chol) and other biochemical products were obtained from Sigma Co. (St. Louis, USA). Phospholipids were washed with acetone containing an antioxidant, $N$-phenyl-p-phenylenediamine, at $4^{\circ} \mathrm{C}$ overnight ${ }^{7}$ and dried under a stream of nitrogen.

Membrane currents under a voltage clamp were recorded with a current amplifier Model L/M EPC-7 (List Electronic, Darmstadt, FRG). The electrical stimulus was applied with a function generator PAR 175 (Princeton Applied Research, Princeton, USA).

\section{Preparation of glutamate binding protein}

The brains from 6-8 adult male rats were used to obtain synaptic membranes. The procedures for the preparation of synaptic plasma membranes have been described in detail in a previous paper. ${ }^{8}$ Synaptic membrane pellets were solubilized with a $2 \%$ Triton X100 solution that contained $0.4 \mathrm{mg} / \mathrm{ml} \mathrm{dog}$ brain PE and $0.1 \mathrm{mg} / \mathrm{ml}$ Chol in a sucrose buffer $(0.25 \mathrm{M}$ sucrose, $10 \mathrm{mM}$ potassium acetate, $0.1 \mathrm{mM}$ EDTA, $0.1 \mathrm{mM}$ dithiothreitol, $10 \%$ glycerol, $10 \mathrm{mM}$ Tris- $\mathrm{HCl}, \mathrm{pH} 7.45$ ). The glutamate binding protein (GBP) from the solubilized membrane extract was purified by affinity batch separation on glass fibers with co-reticulated L-glutamate. The GBP fraction was eluted from the glass fibers with $5 \mathrm{mM} \mathrm{L-Glu}$ and the lipids in sucrose buffer solution that contained $0.1 \% n$-octylglycoside instead of 
Triton X-100. The eluate was dialyzed against the buffer solution $(20 \mathrm{mM}$ potassium acetate, $0.1 \mathrm{mM}$ EDTA, $0.1 \mathrm{mM}$ dithiothreitol, $10 \%$ glycerol, $10 \mathrm{mM}$ Tris- $\mathrm{HCl}$, pH 7.45) to remove L-Glu and $n$-octylglycoside. The proteins in the solution obtained from the glass fiber affinity batch separation were concentrated by using a polyvinyl pyrrolidine bed. The concentrated solution was loaded on a Bio-Beads SM2 column and incubated in order to completely remove the detergent. The protein-liposomes obtained following the elution of the Bio-Beads column were centrifuged at $300000 \mathrm{~g}$ for $1 \mathrm{~h}$ in a Beckman Type-65 rotor. The pelleted material represented the protein reconstituted liposome preparation and was resuspended in a saline solution $(150 \mathrm{mM}$ $\mathrm{NaCl}, 0.2 \mathrm{mM} \mathrm{CaCl}, 5 \mu \mathrm{M}$ glycine, $5 \mu \mathrm{M} \mathrm{MgCl}, 5 \mathrm{mM}$ Hepes/ $\mathrm{NaOH}, \mathrm{pH} 7.5$ ). The concentration of GBP in the reconstituted liposome solution was $c a .8 \times 10^{-8} \mathrm{M}$. The protein solution was stored under argon at $4^{\circ} \mathrm{C}$ in order to prevent oxidation of the lipid.

\section{Fabrication of the receptor ion-channel test sensor}

Two approaches were employed in testing the viability of the lipid bilayer reconstituted glutamate RIC protein as a sensor. These were the single-channel patch-clamp ${ }^{9}$ and the multi-channel Takagi-Montal method. ${ }^{10-12}$ That is, only a single GBP is incorporated in the bilayer lipid membrane (BLM) which is attached at the tip of a $1 \mu \mathrm{m}$ opening of a glass capillary. In the case of the multichannel sensor, several glutamate RIC proteins are incorporated into a much larger two-dimensional area of the BLM.

\section{Procedure to form single channel test sensor}

Patch pipets were made from glass capillary tubes (Fisher Co., Microhematocrit capillary tubes, Blue coded tip, USA) by using a vertical pipet puller (ListElectronic, Model 3P-A, FRG). The capillary was pulled in two stages. ${ }^{9}$ In the first pull the current setting for the heating element was at $20 \mathrm{~A}$ with a pulled length of $9 \mathrm{~mm}$. The thinnest part of the capillary was recentered by using a spacer disk after $1 \mathrm{~min}$. The second pull was made with a current of $12.5 \mathrm{~A}$. These conditions produced a capillary with a tip diameter of $c a .1 \mu \mathrm{m}$.

The pipet was filled by drawing solution through the tip by suction and the backfilling with a syringe. This pipet with an inner filling solution was set on a pipet holder. The BLM was formed on the tip of the pipet, basically, from monolayers of the lipid at the air-water interface. ${ }^{13,14}$ About $400 \mu$ l of the protein reconstituted liposome solution was poured into the Teflon chamber and allowed to stand for $15 \mathrm{~min}$ so that a monolayer would form with the GBP on the surface of the solution. The pipet, which was mounted on the holder of a micromanipulator (Narishige, Model MG-103, Tokyo, Japan), was lowered and vertically immersed twice into the solution. During the second immersion, the BLM was formed incorporating the glutamate RIC protein on the tip of the pipet.

It has been known that the formation of the BLM on the tip of the pipet can be confirmed by measuring the change in the electrical resistance. That is, the resistance is on the order of gigaohms when the tip is completely sealed by the BLM in the patch-clamp method. ${ }^{14}$ The resistance can be measured by the application of a square-wave voltage pulse between the two reference electrodes; one inserted into the upper part of the patchclamp capillary and the other into the bulk solution in which the tip of the capillary is immersed. An "open" unclamped tip had a resistance of ca. $5 \mathrm{M} \Omega$, whereas a sealed pipet tip had a resistance in the order of G $\Omega$.

In order to verify that this lipid membrane on the tip of the pipette was actually a bilayer rather than a multilayer, gramicidine was added into the inner filling and bathing solution. Gramicidin is known to be a pore former "active" only in a bilayer and not in a multilayer. Thus, the conductance change observed after gramicidin was added at an applied constant potential of $80 \mathrm{mV}$ was similar to those reported for other bilayer systems under similar conditions. ${ }^{14}$ Therefore, it can be concluded that an unilamellar bilayer was formed at the tip of the $1 \mu \mathrm{m}$ diameter capillary.

It has been reported that a "tight" BLM should produce only small noise fluctuations, which is a necessary condition to measure ion-channel currents of the order of a few picoamperes. ${ }^{9}$ Experimentally, the probability of obtaining a tight, stable bilayer appeared to be when the pipet was held vertically against the lipid monolayer throughout the entire process of the patchclamp measurements. It is important that vibrations be kept to a minimum for bilayer stability. Most stable bilayers were obtained from $10 \mathrm{mg} / \mathrm{ml}$ of lipid suspension in the case of $\mathrm{PE}$ : Chol mixture with a weight ratio of $4: 1$.

Because the resting potential of biological cell membranes is known to be $60-100 \mathrm{mV}$, the applied potential across the bilayer lipid membrane was kept at $80 \mathrm{mV}$ in the present experiment.

Several molecules of GBP were assumed to be present in the tip area of the pipet, based on an estimation using the values of the protein concentration and the amount of lipids in the solution (vide supra). However, the response of the sensor formed with the abovementioned method showed a characteristic single-step square-wave signal (vide infra). Thus, just a single molecule of GBP was supposed to be present, or at least active, in the BLM on the tip of the pipet. The ability to record single receptor channel currents was about $10 \%$ of all the seals.

\section{Multi-channel test sensor with Takagi-Montal method}

The multi-channel glutamate test sensor was fabricated using the Takagi-Montal method. A small aperture of about $200 \mu \mathrm{m}$ diameter was formed on a thin (12.5 $\mu \mathrm{m}$ thick) Teflon film (YSI membrane kit No. 5776, Yellow Spring, OH, USA) by an electrically sharpened 27 gauge needle. ${ }^{15}$ The Teflon film was tightly sealed vertically between two Teflon chambers. A $2 \%$ $(v / v)$ hexadecane in hexane was painted around the 
aperture and dried under nitrogen stream to make the Teflon surface hydrophobic. After setting two $\mathrm{Ag} / \mathrm{AgCl}$ reference electrodes into each chamber, $0.75 \mathrm{ml}$ of a saline solution and protein reconstituted liposome solution were filled into each respective chamber.

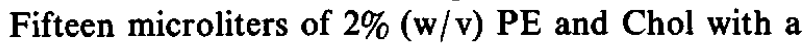
weight ratio of $4: 1$ in hexane was spread onto the saline solution. It required 5-15 $\mathrm{min}$ to remove the hexane by evaporation. A lipid monolayer was formed on top of the saline solution. Next, the water levels in the two chambers were gradually and synchronously raised by adding more solution slowly with a pair of syringes through external tubings. The flow rate was controlled at $50 \mu \mathrm{l} / \mathrm{min}$ with a syringe pump (Harvard Apparatus, Compact Infusion Pump Model 973, MA, USA). Monolayers were readily folded to planar bilayers containing the ion channel protein by raising the water levels, successively, above the aperture. ${ }^{15}$ The final volume of each solution in the chamber was $1.5 \mathrm{ml}$. The tubes for the solution addition into the chamber were disconnected after the formation of the BLM, because they increased the level of electrical noise inductively carried from outside the Faraday cage.

The formation of a sealed planar bilayer membrane (BLM) on the Teflon aperture was confirmed using the same procedure as was used for the single-channel patchclamp. The capacitance, determined from the charging current when a square pulse was applied to the planar lipid membrane in the aperture of $200 \mu \mathrm{m}$ diameter, was between 0.12 and $0.20 \mu \mathrm{F} / \mathrm{cm}^{2}$. Such planar lipid membranes remained stable for more than $24 \mathrm{~h}$ if the entire system was maintained vibration free.

\section{Results and Discussion}

Concentration dependence of the reconstituted glutamate receptor ion-channel

It is essential that the sensing element of a chemical sensor, such as one based on a receptor ion-channel, transduces chemical signals into electrical ones and responds to the analyte in a known reproducible fashion.
Thus, it was important to determine the characteristics of the reconstituted glutamate receptor ion-channel in the two proposed configurations: single-channel patchclamp and the multi-channel method. Figure 1 shows the observed current response of the single ion-channel sensor. No signal was observed in the control experiment where no glutamate receptor ion-channel protein was incorporated. The "on" signal corresponds to the L-glutamate interacting with the receptor to "open" the channel. The signal is thus digital in nature with distinct "on" and "off" or "yes" and "no" states.

An important property of a sensor based on a receptor ion-channel is the built-in function of signal amplification. That is, ions are transferred through the channel during the open time, which is then translated to a measurable current. Each pulse signal from a single channel opening is the result of an active site on the receptor ion-channel interacting (binding) with one or more stimulus analyte molecules. Details in terms of whether the opening relies on a cooperative interaction involving more than one glutamate or glutamate with glycine, for example, are currently unknown. However, assuming that the channel opening requires only a single L-glutamate interacting with the receptor, the amplification factor will be defined as the total number of ions transported across the open channel during the "on" state. In order to calculate this factor, the mean open time and the channel currents need to be known. The data from experiments, such as that shown in Fig. 1, were analyzed in order to obtain the pulse height associated with the open times, as indicated in Fig. 2. As may be seen in this figure, the pulse heights are essentially independent of the channel open times. The average current was $4.45 \mathrm{pA}$ for an applied potential of $80 \mathrm{mV}$ across the BLM. When the number of events at the same open time was plotted versus the open time, the plot shown in Fig. 3 was obtained. The number of openings decreased nearly exponentially with the open time. For a total of 161 openings, the mean open time was calculated to be $7.6 \pm 3.8 \mathrm{~ms}$. The number of sodium ions transported through the ion-channel during this average open time was 210000. An amplification factor of

A

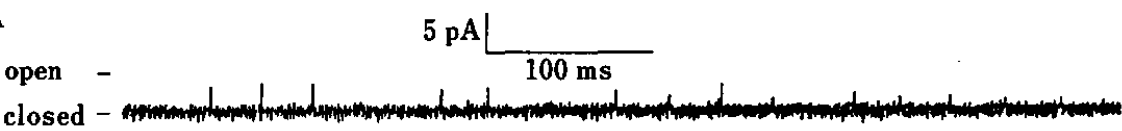

B

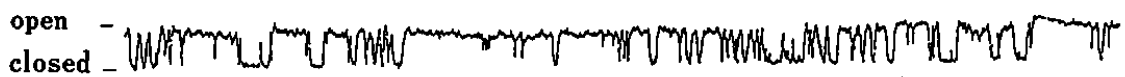

Fig. 1 Response of a single-channel test sensor. Inner filling solution: $150 \mathrm{mM} \mathrm{NaCl}, 0.2 \mathrm{mM} \mathrm{CaCl}, 5 \mu \mathrm{M} \mathrm{MgCl}, 5 \mu \mathrm{M}$ glycine, $25 \mu \mathrm{g} / \mathrm{ml}$ concanavaline A, $5 \mathrm{mM}$ Hepes/ $\mathrm{NaOH}$, pH 7.5, containing A) $50 \mu \mathrm{M}$ and B) $500 \mu \mathrm{M}$ of L-Glu. Bathing solution contains GBP/lipid suspension in the same composition as the inner filling solution except for concanavaline $A$ and L-Glu. The applied potential was $80 \mathrm{mV}$. 


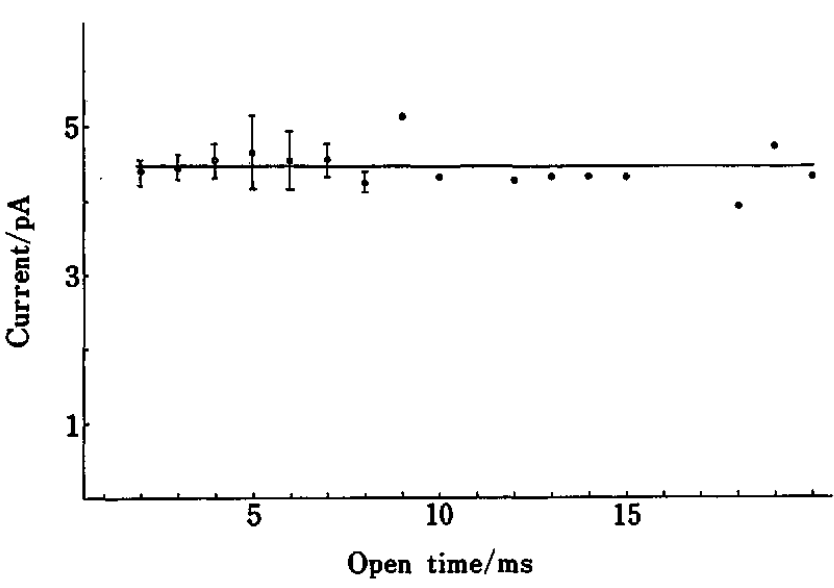

Fig. 2 Distribution of the signal height associated with the open time analyzed from the data of pulse signals in Fig. 1(B). Values represent the mean of events at the same open time. Variation bars correspond to the standard deviation for events.

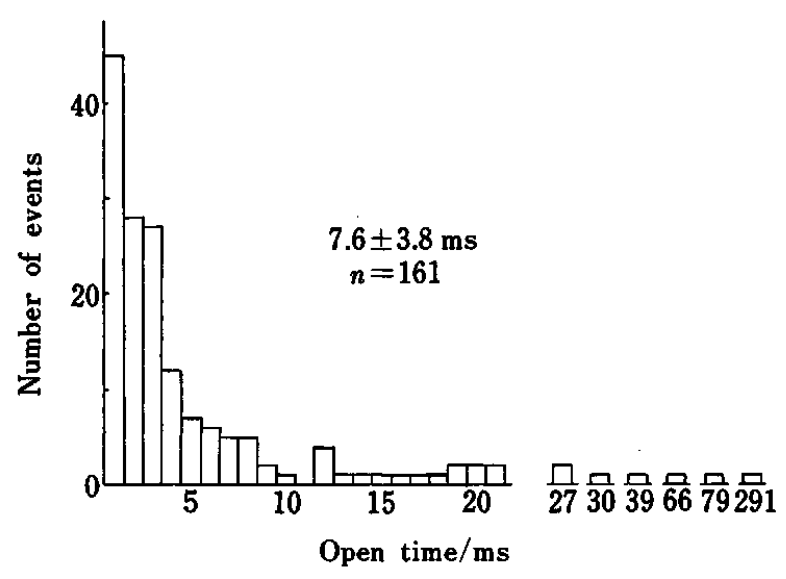

Fig. 3 Distribution of events that had the same open time, analyzed from the data of pulse signals in Fig. 1(B).

210000 was calculated on the assumption that the channel is opened by one glutamate molecule.

Although data are limited, similar analyses were made by us for other single-channel recordings from the literature ${ }^{17,18}$ and the amplification factors are summarized in Table 1 together with our value. With L-Glu receptor ion-channel obtained directly from a locust muscle fiber, an amplification factor of 186000 was obtained. For the acetylcholine RIC stimulated with $10 \mu \mathrm{M}$ of acetylcholine, amplification factors were calculated to be $\mathbf{1 5 0 0 0}$ to as large as 95000 .

The concentration dependence of the channel openings was then examined. For 50 and $500 \mu \mathrm{M}$ Lglutamate, pulse frequencies of 23 and $100 \mathrm{~Hz}$, respectively, were observed. With $10^{-3} \mathrm{M}$ of L-Glu concentration, the observed current responses showed a striking pattern with an unstable and much larger amplitude
Table 1 The amplification factors obtained with some receptor ion-channel proteins

\begin{tabular}{lcccc}
\hline Stimulant/ $\mu \mathrm{M}$ & $\begin{array}{c}\text { Mean open } \\
\text { time/ms }\end{array}$ & $\begin{array}{c}\text { Channel } \\
\text { current/pA }\end{array}$ & $\begin{array}{c}\text { Amplification } \\
\text { factor }\end{array}$ \\
\hline L-Glu $^{\mathrm{a}}$ & 500 & 7.6 & 4.45 & 210000 \\
L-Glu $^{\mathrm{b}}$ & 500 & 6.5 & 4.6 & 186000 \\
Ach $^{\mathrm{c}}$ & 10 & 4.5 & 3.4 & 95000 \\
& & & 0.7 & 15000 \\
\hline
\end{tabular}

The amplification factor, $\mathrm{A}$, was defined by assuming that the number of the stimulant needed to open the channel is unity. $A=($ mean open time $) \times($ channel current $) \times($ Avogadro's number) $/ 96500$.

a. The conditions the same as in Fig. 1B).

b. From reference 17 .

c. From reference 18. Ach; acetylcholine. An applied potential of $15 \mathrm{mV}$ was used. The value of the channel current was corrected for at an applied potential of $80 \mathrm{mV}$ for a comparison with our data.

than that of signals shown in Fig.1. The responses were different from those of the characteristic digital signals as shown in Fig. 1. They were, thus, excluded from the data analysis described earlier (Figs. 2, 3). Irrespective of our earlier assumption to define the amplification factor, the on and off states do not appear to be dependent on the binding of a single glutamate molecule, but more likely involves a series of cooperative steps. However, the overall probability of the channel opening appears to be concentration dependent.

Although data are still very much limited, because of the experimental difficulties in single-channel successes, the observed frequency $(f)$ appears to follow an empirical relationship,

$$
f=1 /(A+B)
$$

where $A$ is the mean open time and $B$ the mean closed time of the pulse signals from the single channel recording (see Fig. 1). The $A$ term becomes important at higher concentrations whereas the value of the $B$ term decreases. Thus, a plot of the frequency versus concentration is non-linear, approaching what appears to be saturation at high concentrations. Since the potentiality of the present single ion channel sensor is most likely for the detection of extremely low concentrations of analyte, the $A$ term will be essentially negligible in this concentration range of interest. In the case of the multi-channel sensor, the area of the "sensing" membrane is about $\mathbf{4 0 0 0 0}$ times larger than that of the single-channel case. Thus, a much greater number of RIC proteins are incorporated into the lipid bilayer. The number of channel openings $(n)$ again depends on the concentration of the interacting analyte. Even if each single RIC in this larger-area bilayer produces a pulse-like signal, the total output signal observed will be a composite of all the RIC proteins. When $n$ is large, this signal will no longer be comprised 


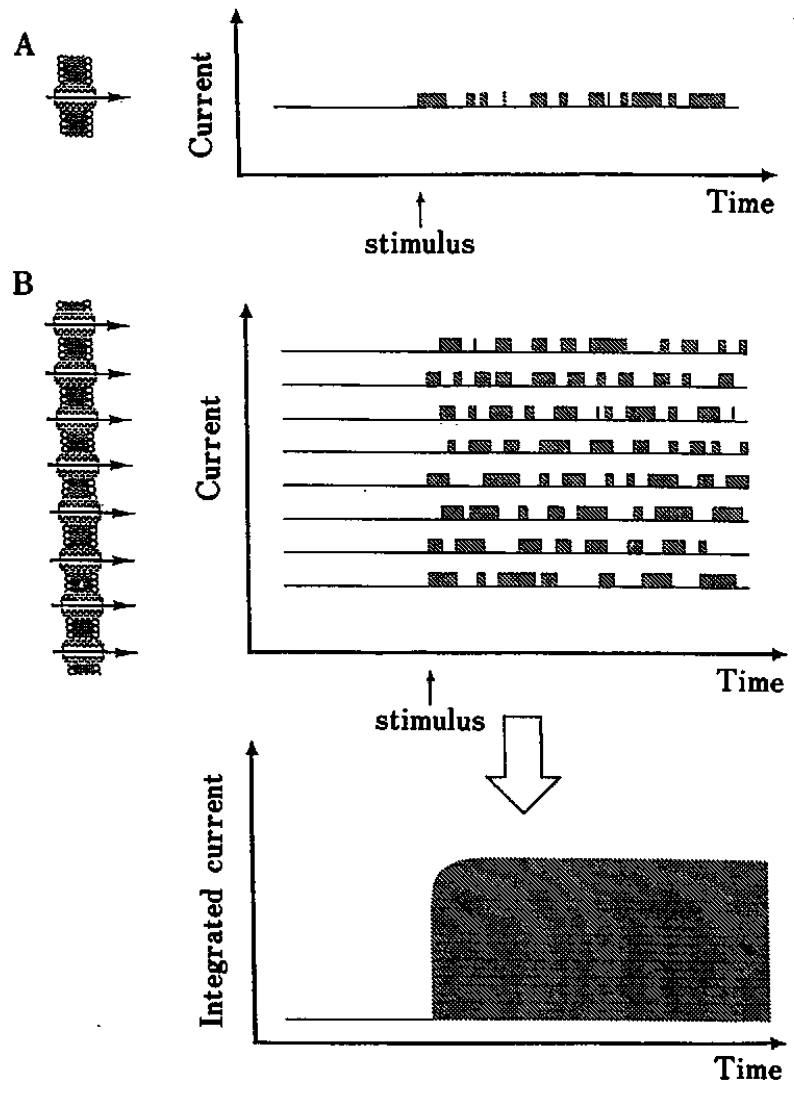

Fig. 4 Schematic representation of the responses of receptor ion-channel test sensors; A) single-channel test sensor and B) multi-channel test sensor.

of discrete pulses but will be analog in nature (dc current), derived from the sum of all pulses. The single- and multi-channel cases are illustrated in Fig. 4. Only as the limit of detectability is approached at low concentrations does the multi-channel signal become similar to that of the single channel. Data to date for the multi-channel glutamate RIC indicate that the steady-state dc current is proportional to the concentration of L-Glu in the concentration range of $50-200 \mu \mathrm{M}$.

\section{Conclusion}

The present study represents the first measurement of the ion-channel current with purified glutamate receptor ion-channel protein, opening the prospect for the development of novel biosensors with an ability of high signal amplification.

The single-channel test sensor recognized glutamate ion as a chemical signal, and transduced it to an electrical signal with an amplification factor of 210000 . The concentration of the analyte can be determined from the frequency of the pulse signal in the single-channel sensor and from a current intensity in the multi-channel sensor. In the present study, a concentration of $10^{-5} \mathrm{M}$ order was examined. With an enzyme electrode using glutamate-dehydrogenase ${ }^{19}$ or a microorganism sensor using Escherichia coli ${ }^{20}$, the limit of detection for L-Glu concentration is about $7 \times 10^{-5} \mathrm{M}$. Therefore, the sensitivity of the present receptor ion-channel sensors is quite comparable to that of other sensors even though the measurement conditions have not been optimized. Consequently, the present study has demonstrated the remarkable potentiality of using receptor ion-channel proteins for a new type of chemical- or bio-sensor.

This research was supported in part by the Japan-U. S. Cooperative Sciences Program sponsored by the Japan Society for the Promotion of Science and the U. S. National Science Foundation. The support provided by grants DAAL 03-88K0017 from ARO and CHE-8515663 from NSF is also gratefully acknowledged.

\section{References}

1. P. Yager, Biosensors, 2, 363 (1987).

2. A. W. Dalziel, J. Georger, R. P. Price, A. Singh and P. Yager, "Membr. Proteins, Proc. Membr. Protein Symp. 1986", ed. S. C. Goheen, p. 643, Bio-Rad Lab., Richmond, California, 1987.

3. M. Gotoh, E. Tamiya, M. Momoi, Y. Kagawa and I. Karube, Anal. Lett., 20, 857 (1987).

4. M. E. Eldefrawi, S. M. Sherby, A. G. Andreou, N. A. Mansour, Z. Annau, N. A. Blum and J. J. Valdes, Anal. Lett., 21, 1665 (1988).

5. H. G. Smith, Abstract for "Symposium on Agents of Biological Origins", Johns Hopkins University, March 1989.

6. R. F. Taylor and I. G. Marenchic, E. J. Cook, Anal. Chim. Acta, 213, 131 (1988).

7. Y. Kagawa and E. J. Racker, J. Biol. Chem., 246, 5477 (1971).

8. J.-W. Chen, M. D. Cunningham, N. Gulton and E. K Michaelis, J. Biol. Chem., 263, 417 (1988).

9. B. Sakmann and E. Neher, "Single-Channel Recording", Plenum Press, New York, 1983.

10. M. Takagi, "Seitaimaku Jikkengïutu (Experimental Techniques in Biomembrane Research, in Japanese)", ed. T. Ohnishi, p. 385, Nankodo, Tokyo, 1967.

11. M. Montal and P. Mueller, Proc. Natl. Acad. Sci. USA, 69, 3561 (1972).

12. C. Miller, Physiol. Rev., 63, 1209 (1983).

13. B. A. Suarez-Isla, K. Wan, J. Lindstorm and M. Montal, Biochem., 22, 2319 (1983).

14. R. Coronado and R. Latorre, Biophys. J., 43, 231 (1983).

15. C. Miller, "Ion Channel Reconstitution", Plenum Press, New York, 1986.

16. M. Schindler and U. Quast, Proc. Natl. Acad. Sci. USA, 77, 3052 (1980).

17. K. A. F. Gration, J. J. Lambert, R. L. Ramsey, R. P. Rand and P. M. R. Usherwood, Brain Res., 230, 400 (1981).

18. P. Labarca, M. S. Montal, J. M. Lindstorm and M. J. Montal, Neurosci., 5, 3409 (1985).

19. I. Karube, Maku, 7, 332 (1982).

20. I. Hikuma, H. Obana, T. Yasuda, I. Karube and S. Suzuki, Anal. Chim. Acta, 116, 61 (1980). 